\title{
Particle transport in a network of quantum harmonic oscillators
}

\author{
J. R. Yusupov ${ }^{1}$, Kh. Sh. Matyokubov ${ }^{2}$, K. K. Sabirov ${ }^{3}$ \\ ${ }^{1}$ Turin Polytechnic University in Tashkent, 17 Niyazov Str., 100095, Tashkent, Uzbekistan \\ ${ }^{2}$ Urgench State University, 14 H. Olimjon Str., 220100, Urgench, Uzbekistan \\ ${ }^{3}$ Tashkent University of Information Technologies, 108 Amir Temur Str., 100200, Tashkent Uzbekistan \\ jambul.yusupov@gmail.com
}

PACS 03.65.Aa, 02.60.Cb, 05.60.Gg

DOI 10.17586/2220-8054-2020-11-2-145-152

\begin{abstract}
In this paper, we address the problem of a particle dynamics in a network of quantum harmonic oscillators by solving the stationary Schrödinger equation on metric graphs in the presence of harmonic oscillator potential with bond-dependent frequency. Particle transport is analyzed by considering wave packet dynamics in such a system modeled in terms of quantum graph.
\end{abstract}

Keywords: quantum graph, harmonic oscillator, particle transport, wave packet dynamics.

Received: 13 February 2020

Revised: 24 February 2020

\section{Introduction}

Quantum transport in low-dimensional branched structures and networks is of importance for a broad variety of problems in condensed matter physics, polymers, optics and biophysics. For of sub-micron scales, particle and wave dynamics in networks can be described in terms of so-called quantum graphs. The latter is a set of quantum wires connected to each other at the nodes (vertices) according to a rule, which is called the topology of a graph. Recently quantum graphs have attracted much attention in the contexts as powerful model for studying quantum transport in low-dimensional branched systems. Initially, quantum mechanical description of particle transport in networks dates back to Refs. [1,2], where electron motion in organic molecules was studied. Strict formulation of quantum graph concept was first introduced by Exner, Seba and Stovicek to describe free quantum motion on branched wires [3]. Later, Kostrykin and Schrader derived the general boundary conditions providing self-adjointness of the Schrödinger operator on graphs [4]. Earlier, the quantum graph concept was applied to microwave optical fiber networks [5], quantum chaos theory [6-8] and condensed matter physics [9, 10]. Relativistic quantum mechanics described by Dirac [11] and Bogoliubov-de Gennes operators [12] on graphs have been studied recently. Very recent application of quantum graphs to optical network lasers (LANER) problem can be found in the Ref. [13]. The problem for designing of transparent quantum graphs has been discussed in $[14,15]$. A version of PT-symmetric quantum graph was introduced in [16]. Tunable transport in quantum graphs has been studied in $[17,18]$. Different aspects of the linear and nonlinear wave equations on metric graphs are considered in the Refs. [19-28].

Here, we address the problem of a particle dynamics in a network of quantum harmonic oscillators by solving the stationary Schrödinger equation on metric graphs in the presence of harmonic oscillator potential with bond-dependent frequency. To solve Schrödinger equation on harmonic oscillator network we impose for confined harmonic oscillator wave functions the vertex boundary conditions providing continuity of wave function and Kirchhoff rule at the vertex. Such boundary conditions lead to spectral equations in the form of transcendental algebraic equation.

Network of quantum harmonic oscillators can be used for modeling of vibrations and oscillations in discrete or branched systems, such as crystal lattices, molecular chains, polymers, etc. $[5,9,10]$. Also, since crystal lattices can be considered as branched structures, one can use quantum harmonic oscillator network for modeling lattice vibrations in solid state. Our approach for the study of quantum harmonic oscillator network is based on considering it as a set of confined harmonic oscillators connected at vertices. Confined, or bounded harmonic oscillator presents parabolic potential given at finite interval (see, Fig.1). Quantum mechanical bounded oscillator was studied earlier in detail in the Refs. [29-31]. Main motivation for the study of network of quantum harmonic oscillators comes from their potential applications in the physics of conducting polymers, where polymer chain (e.g., in polyacetylene) can be considered as a branched structure, where lattice of the chain forms graph. Charge carriers in such structure are localized (trapped) near one bond of the lattice. Depending on the type of exciton the trapping potential well can be different. For all cases, such a well has a cutoff near the branching point. If one also considers lattice vibrations, most realistic form of the well can be in the form of harmonic oscillators. Thus, exciton moving along the polymer chain behaves itself as a particle moving in the quantum harmonic oscillator network. 


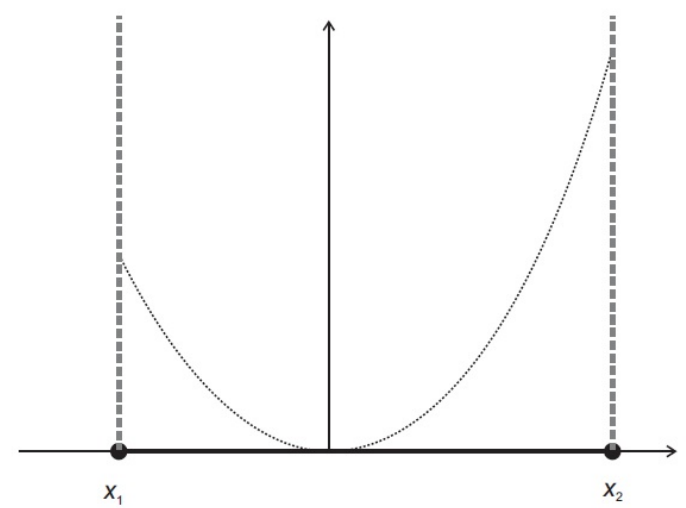

FIG. 1. Parabolic potential given at finite interval

This paper is organized as follows. In the next section, we will provide a brief description of the corresponding problem on a finite interval, i.e., particle dynamics in confined quantum harmonic oscillator potential well. Section 3 presents description of the main problem, quantum harmonic oscillator network. Section 4 provides some concluding remarks.

\section{Confined quantum harmonic oscillator}

Before discussing harmonic oscillator network, we briefly recall the problem of confined quantum harmonic oscillator described by harmonic oscillator potential on a finite interval. Consider a quantum particle confined in a 1D box $\left[x_{1}, x_{2}\right]$ under influence of harmonic potential. Such potential represent parabolic well with the cut-off on the box walls. The Hamiltonian of the system can be written as (in the units $\hbar=m=1$ ):

$$
H=-\frac{1}{2} \frac{d^{2}}{d x^{2}}+\frac{1}{2} \omega^{2} x^{2}
$$

Complete solution of the time-independent Schrödinger equation

$$
H \phi=E \phi
$$

can be written in terms of the confluent hypergeometric function of the first kind:

$$
\phi(x)=\exp \left(-\frac{1}{2} \omega x^{2}\right)\left[A M\left(\frac{1}{4}-\frac{E}{2 \omega} ; \frac{1}{2} ; \omega x^{2}\right)+B \omega^{\frac{1}{2}} x M\left(\frac{3}{4}-\frac{E}{2 \omega} ; \frac{3}{2} ; \omega x^{2}\right)\right],
$$

where $A$ and $B$ are constants and $M(\cdot ; \cdot ; \cdot)$ is the Kummer's function.

The boundary conditions $\phi\left(x_{1}\right)=\phi\left(x_{2}\right)=0$ result in a system of linear algebraic equations with respect to coefficients $A$ and $B$ :

$$
\mathbf{h}(E)\left(\begin{array}{l}
A \\
B
\end{array}\right)=0
$$

where

$$
\mathbf{h}(E)=\left(\begin{array}{cc}
M\left(\frac{1}{4}-\frac{E}{2 \omega} ; \frac{1}{2} ; \omega x_{1}^{2}\right) & \omega^{1 / 2} x_{1} M\left(\frac{3}{4}-\frac{E}{2 \omega} ; \frac{3}{2} ; \omega x_{1}^{2}\right) \\
M\left(\frac{1}{4}-\frac{E}{2 \omega} ; \frac{1}{2} ; \omega x_{2}^{2}\right) & \omega^{1 / 2} x_{2} M\left(\frac{3}{4}-\frac{E}{2 \omega} ; \frac{3}{2} ; \omega x_{2}^{2}\right)
\end{array}\right) .
$$

Existence of the non-trivial solutions of the above algebraic system leads to the following secular equation with respect to $E$ :

$$
\operatorname{det}(\mathbf{h}(E))=0 \text {. }
$$

Roots of this equation give the eigenvalues of the confined quantum harmonic oscillator. In Fig. 2 the first few eigenvalues obtained in this way are plotted for the parameters $\omega=1, x_{1}=0, x_{2}=5$. It is clear that when the size of the box is very large, the eigenvalues coincide with those of bulk (unbound) harmonic oscillator becoming equidistant. 


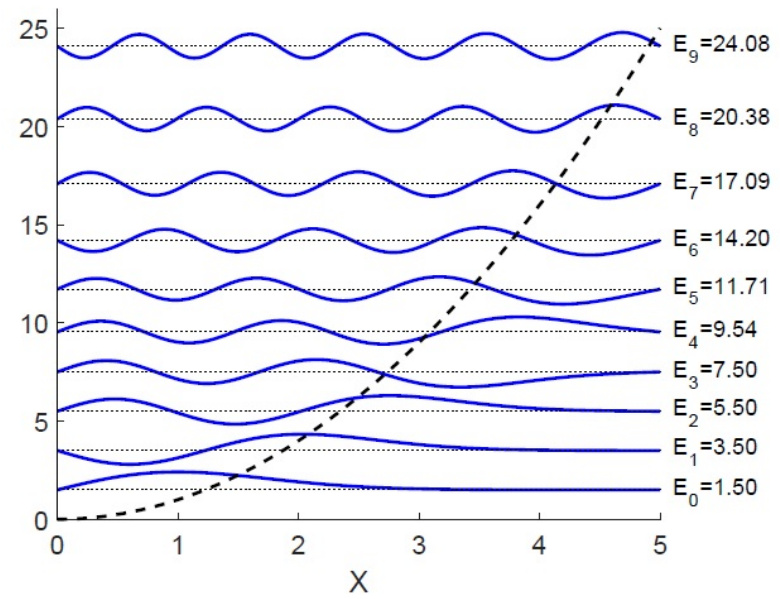

FIG. 2. Oscillator potential (dashed line), energy levels (dotted line) and corresponding eigenfunctions (solid line) for $\omega=1$ and $x \in[0,5]$

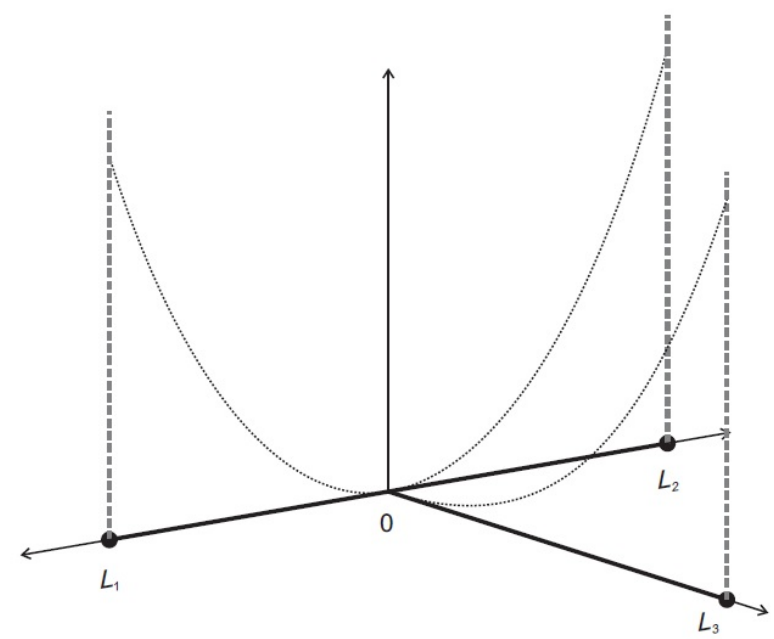

FIG. 3. Considered case: Harmonic oscillators on a star graph with 3 bonds

\section{Harmonic oscillator on a star graph}

The system we are interested to study is a network of quantum harmonic oscillators. Since a star graph can be considered as a building block for any complex networks we limit our study to the simplest graph topology, a star graph presented in Fig. 3. In the absence of external time-dependent forces, such system can be described by the stationary Schrödinger equation on metric graphs that includes harmonic oscillator potential, which is given as (in the units $\hbar=m=1)$ :

$$
\left[-\frac{1}{2} \frac{d^{2}}{d x^{2}}+\frac{1}{2} \omega_{j}^{2} x^{2}\right] \phi_{j}=E \phi_{j}
$$

where $\phi_{j}$ is the wave function of $j$ th bond and (in general) $j=1,2,3, \ldots N$.

The vertex boundary conditions for a star graph are imposed as the wave function continuity [6]:

$$
\left.\phi_{1}\right|_{x=0}=\left.\phi_{2}\right|_{x=0}=\cdots=\left.\phi_{N}\right|_{x=0},
$$

and current conservation [6]:

$$
\left.\sum_{j=1}^{N} \frac{d \phi_{j}}{d x}\right|_{x=0}=0
$$


TABLE 1. The first 20 energy levels of the star graph in Fig. 4

\begin{tabular}{|c|c|c|c|c|c|}
\hline$n$ & 0 & 1 & 2 & 3 & 4 \\
\hline$E_{n}$ & 0.70710 & 2.12132 & 2.12132 & 3.53554 & 4.94981 \\
\hline \hline$n$ & 5 & 6 & 7 & 8 & 9 \\
\hline$E_{n}$ & 4.94993 & 6.36492 & 7.78123 & 7.78573 & 9.21454 \\
\hline \hline$n$ & 10 & 11 & 12 & 13 & 14 \\
\hline$E_{n}$ & 10.65426 & 10.70381 & 12.20934 & 13.74315 & 13.95672 \\
\hline \hline$n$ & 15 & 16 & 17 & 18 & 19 \\
\hline$E_{n}$ & 15.62432 & 17.32891 & 17.82647 & 19.68142 & 21.57984 \\
\hline
\end{tabular}

The boundary conditions at the bond edges can be imposed as Dirichlet ones:

$$
\left.\phi_{j}\right|_{x=L_{j}}=0,
$$

where $L_{j}$ are the bond lengths.

Solution of Eq. (7) can be written as

$$
\phi_{j}(x)=\exp \left(-\frac{1}{2} \omega_{j} x^{2}\right)\left[A_{j} M\left(\frac{1}{4}-\frac{E}{2 \omega_{j}}, \frac{1}{2}, \omega_{j} x^{2}\right)+B_{j} \omega_{j}^{1 / 2} x M\left(\frac{3}{4}-\frac{E}{2 \omega_{j}}, \frac{3}{2}, \omega_{j} x^{2}\right)\right],
$$

where $A_{j}$ and $B_{j}$ are the constants to be found from normalization conditions and $M(\cdot ; \cdot ; \cdot)$ is the confluent hypergeometric function. From the boundary conditions given by Eqs. (8)-(10) one can find energy eigenvalues, $E_{n}$ and constants, $A_{j}$ and $B_{j}$.

The continuity condition (8) leads to:

$$
A_{1}=A_{2}=\cdots=A_{N}:=A .
$$

From the current conservation condition given by Eq. (9) we have

$$
\sum_{j=1}^{N} B_{j} \omega_{j}^{1 / 2}=0 .
$$

Finally, from the Dirichlet boundary conditions on the bonds edges, Eq. (10) we get the following system of linear algebraic equations with respect to coefficients $A$ and $B_{j},(j=1,2, \ldots, N)$ :

$$
\mathbf{h}(E)\left(\begin{array}{c}
A \\
B_{1} \\
\vdots \\
B_{N}
\end{array}\right)=0
$$

where

$$
\mathbf{h}(E)=\left(\begin{array}{ccccc}
0 & \omega_{1}^{1 / 2} & \omega_{2}^{1 / 2} & \ldots & \omega_{N}^{1 / 2} \\
\mu_{1} & \eta_{1} & 0 & \ldots & 0 \\
\mu_{2} & 0 & \eta_{2} & \ldots & 0 \\
\vdots & \vdots & \vdots & \ddots & \vdots \\
\mu_{N} & 0 & 0 & \ldots & \eta_{N}
\end{array}\right)
$$

with $\mu_{j}=M\left(\frac{1}{4}-\frac{E}{2 \omega_{j}}, \frac{1}{2}, \omega_{j} L_{j}^{2}\right)$ and $\eta_{j}=\omega_{j}^{1 / 2} L_{j} M\left(\frac{3}{4}-\frac{E}{2 \omega_{j}}, \frac{3}{2}, \omega_{j} L_{j}^{2}\right)$.

Existence of non-trivial solutions of this system yields the following secular equation:

$$
\operatorname{det}(\mathbf{h}(E))=0 .
$$

The first ten eigenfunctions are plotted in Fig. 4 and the first twenty energy levels are presented in Table 1. Calculations have been done for $N=3$ and parameters $\omega_{1}=\omega_{2}=\omega_{3}=1$ and $L_{1}=5.31, L_{2}=5.53, L_{3}=5.18$. Bond lengths of the star graph have been chosen such in order to avoid degeneracy in numerical calculations. Although the energy grows by growing of $n$, unlike to usual harmonic oscillator, the levels are not equidistant. Moreover, the level spacing distances are strongly non-uniform for such a system. 

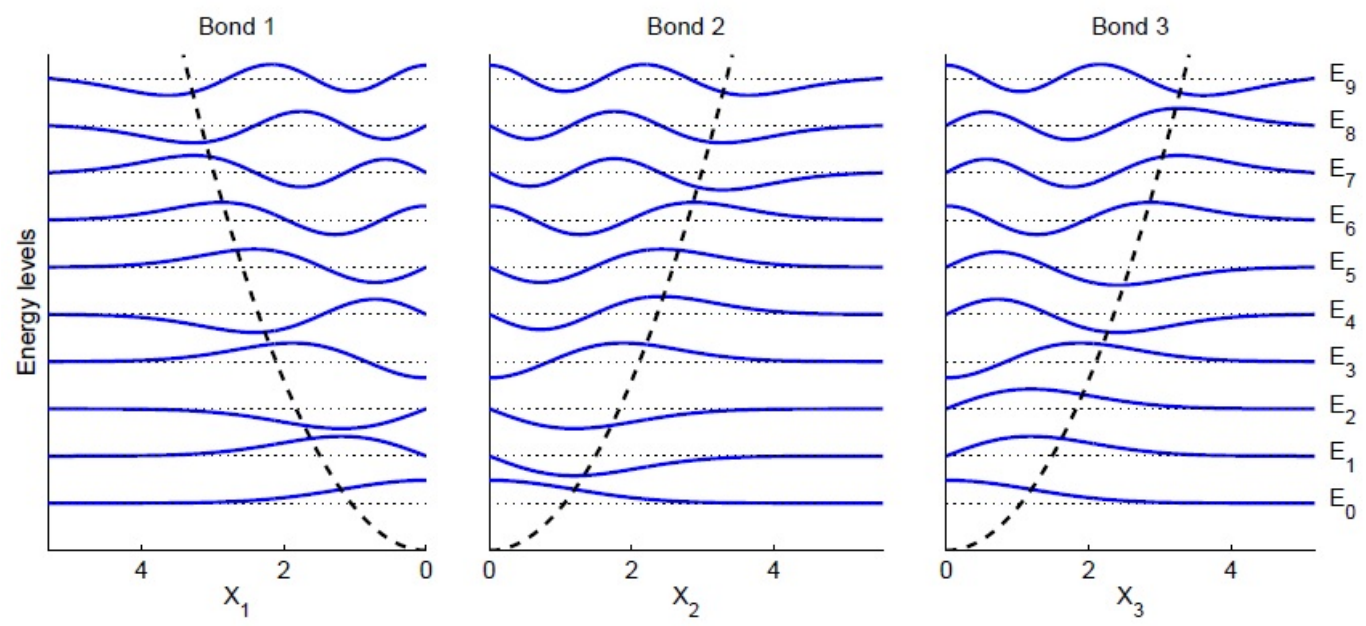

FIG. 4. Oscillator potential (dashed line), energy levels (dotted line) and corresponding eigenfunctions (solid line) for $\omega_{1}=\omega_{2}=\omega_{3}=1$ and $L_{1}=5.31, L_{2}=5.53, L_{3}=5.18$. The three columns correspond to the bonds of the star graph. The $x$-coordinate of the first arm is reversed

Having found eigenfunctions and eigenvalues of such a network, one can study particle transport in it by considering wave packet dynamics. This means that one needs to solve the time-dependent Schrödinger equation (in the units $\hbar=m=1$ ) given as:

$$
i \frac{\partial}{\partial t} \Psi_{j}(x, t)=\left(-\frac{1}{2} \frac{\partial^{2}}{\partial x^{2}}+\frac{1}{2} \omega_{j}^{2} x^{2}\right) \Psi_{j}(x, t), j=1,2,3 .
$$

The wave function of the system can be written in terms of the complete set of eigenfunctions as:

$$
\Psi_{j}(x, t)=\sum_{n} c_{n} e^{-i E_{n} t} \phi_{j, n}(x),
$$

where $\phi_{j, n}(x)$ and $E_{n}$ are given by Eqs. (11) and (16). Wave packet dynamics can be studied by imposing initial condition in the form of Gaussian wave packet given by:

$$
f(x)=\frac{1}{d \sqrt{\pi}} \exp \left[-\frac{\left(x-x_{0}\right)^{2}}{2 d^{2}}+i v_{0} x\right],
$$

and determining expansion coefficients, $c_{n}$ in terms of $f(x)$ as

$$
c_{n}=\sum_{j} \int_{0}^{L_{j}} \phi_{j, n}^{*}(x) f(x) d x .
$$

In the following numerical calculations, we consider quantum star graph with bond lengths $L_{1}=5.31, L_{2}=5.53$, $L_{3}=5.18$ and Gaussian wave packet initially located in the first bond at $x_{0}=L_{1} / 2$ at rest, that is with initial group velocity $v_{0}=0$ and width $d=L_{1} / 10$. This setup can be approximated by choosing expansion coefficients in the form:

$$
c_{n}=\int_{0}^{L_{1}} \phi_{1, n}^{*}(x) f(x) d x .
$$

Then, the numerical solution requires to limit the number of expansion terms. In our calculations, we limit ourselves to 300 terms, which have been enough for controlling the deviation from unity in the normalization condition,

$$
\sum_{n}\left|C_{n}\right|^{2}=1
$$

Thus, space-time evolution of Gaussian wave packet in a network of quantum harmonic oscillators having similar (for all bonds) frequencies is plotted in Fig. 5. Fig. 6 presents similar plots for the case when frequencies of harmonic oscillators are different for different bonds: $\omega_{1}=1, \omega_{2}=2, \omega_{3}=3$. As it can be seen from these plots, dispersion of the wave packet is much stronger in the case of different frequencies. 

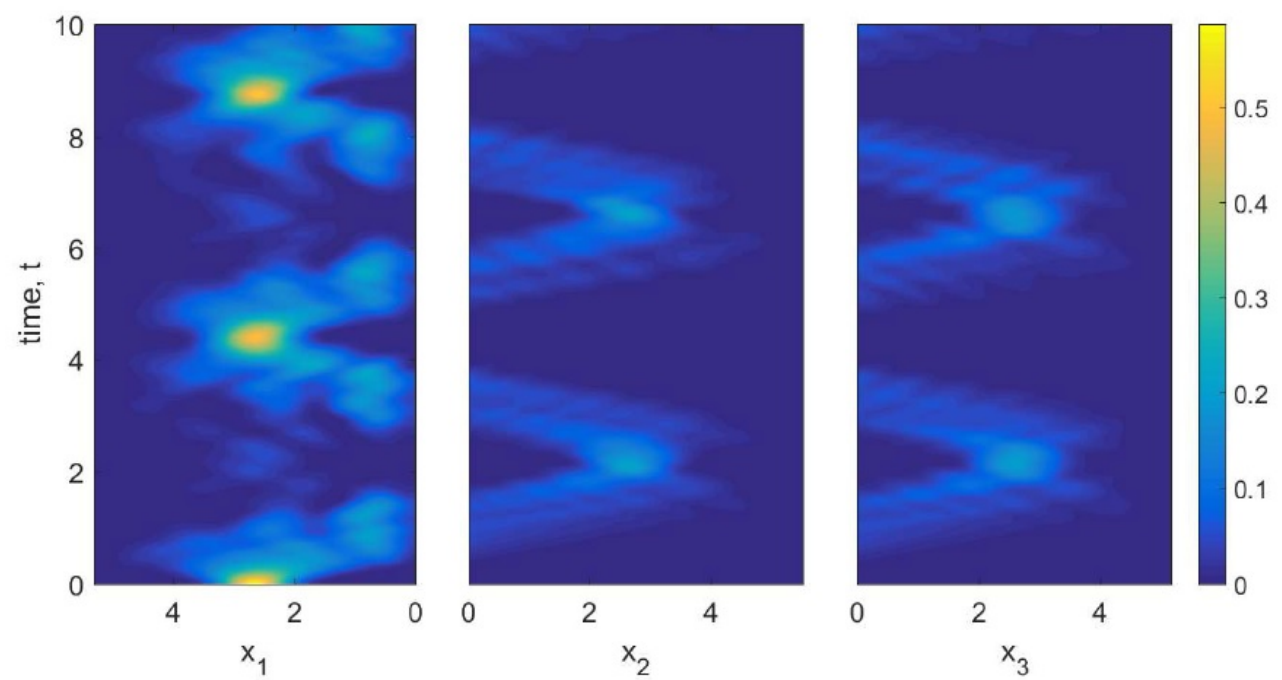

FIG. 5. Space-time evolution of Gaussian wave packet in a network of quantum harmonic oscillators. Frequencies of oscillators in all bonds are equal: $\omega_{1}=\omega_{1}=\omega_{3}=1$
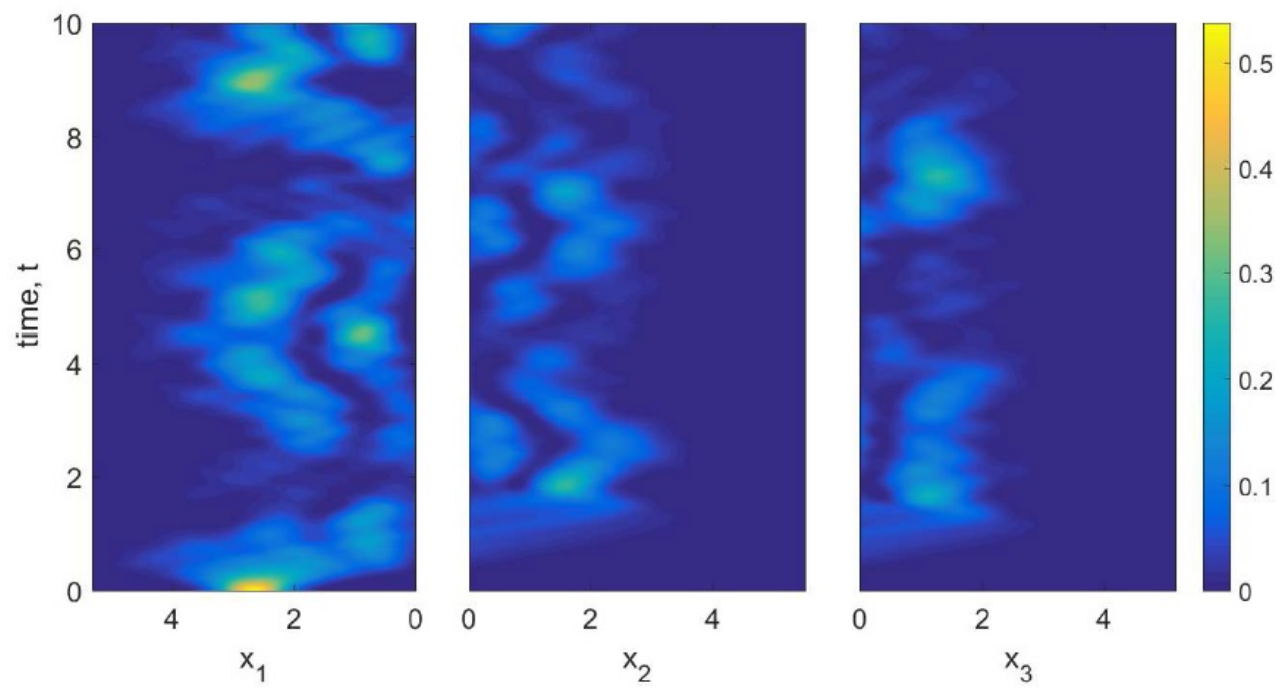

FIG. 6. Space-time evolution of Gaussian wave packet in a network of quantum harmonic oscillators for frequencies, $\omega_{1}=1, \omega_{2}=2, \omega_{3}=3$

An important characteristics of the particle and wave transport in quantum graphs is probability current. Current on each bond of the graph is given by:

$$
J_{j}(x, t)=\frac{i}{2}\left[\Psi_{j}(x, t) \frac{\partial \Psi_{j}^{*}(x, t)}{\partial x}-\frac{\partial \Psi_{j}(x, t)}{\partial x} \Psi_{j}^{*}(x, t)\right] .
$$

In Fig. 7 and Fig. 8 time-dependence of current on each bond is plotted for the cases presented in Fig. 5 and Fig. 6, respectively. 

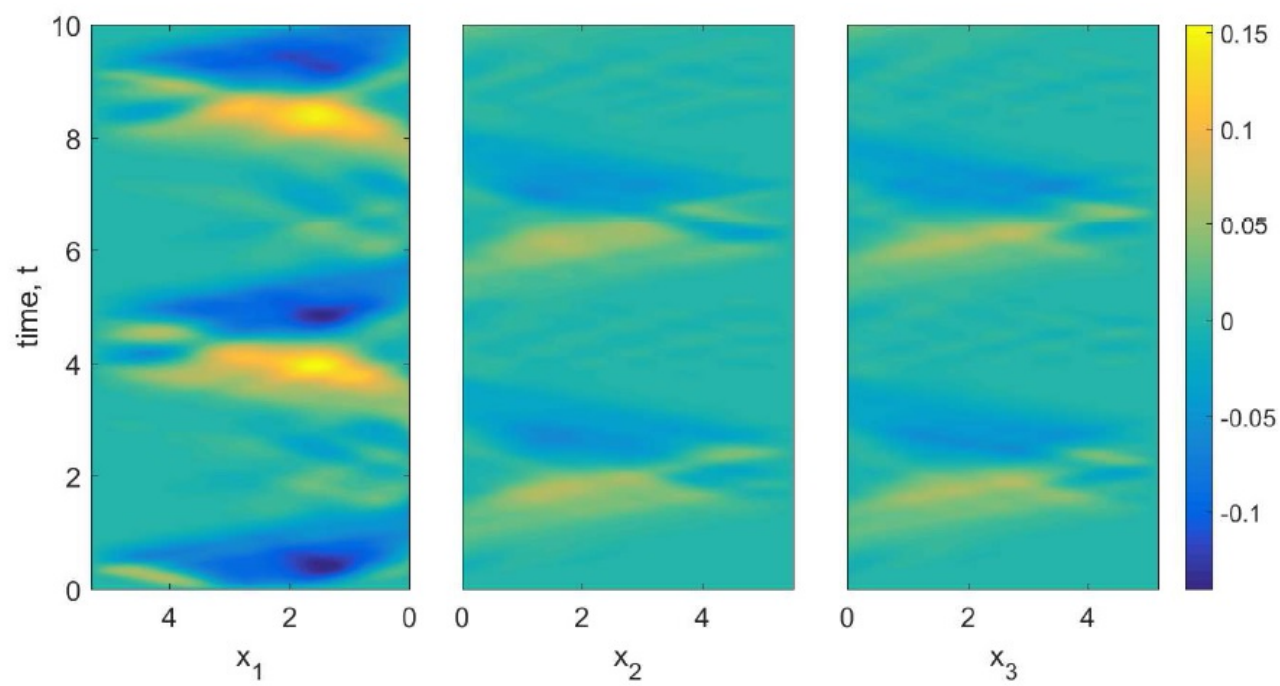

FIG. 7. Time-dependence of the current for equal frequencies, $\omega_{1}=1, \omega_{2}=1, \omega_{3}=1$
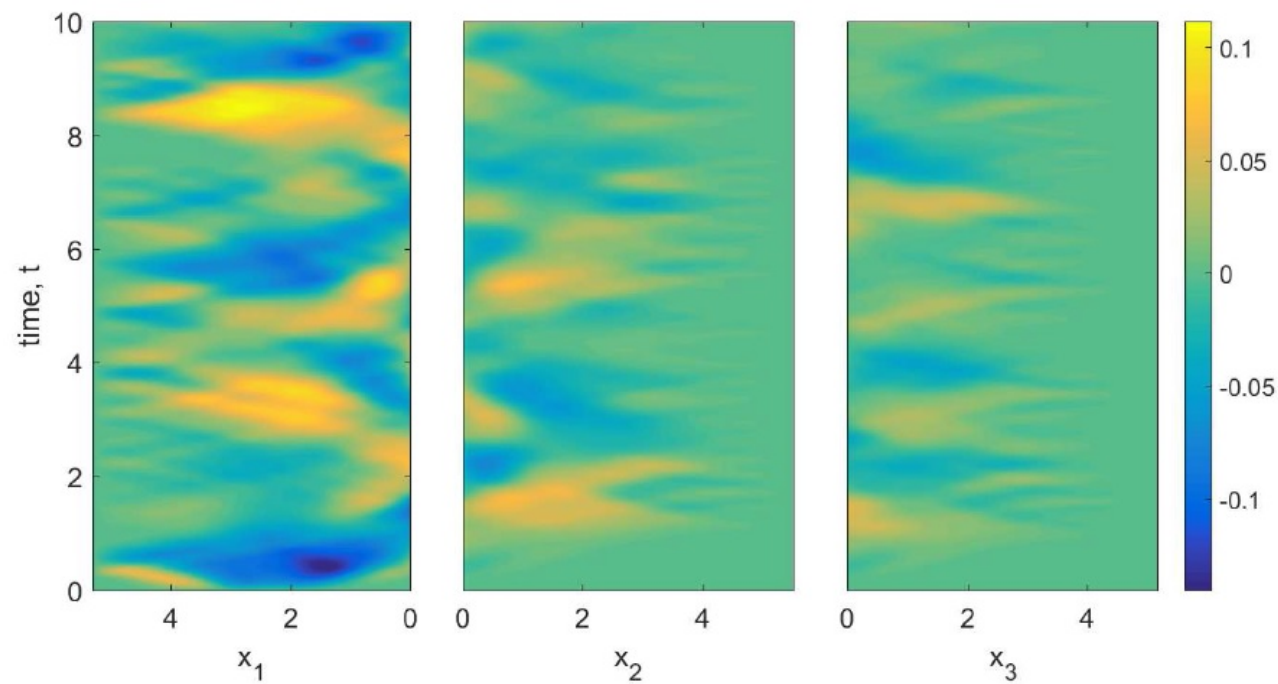

FIG. 8. Time-dependence of the current. Frequencies of oscillators are different: $\omega_{1}=1, \omega_{2}=2$, $\omega_{3}=3$

\section{Conclusions}

In this paper we studied quantum harmonic oscillator network with the focus on energy spectrum and particle transport. The system is modeled in terms of the stationary Schrödinger equation on metric graphs by imposing continuity and current conservation boundary conditions at the graph nodes. The energy spectrum is calculated numerically from the secular equation following from the vertex boundary conditions. Particle transport is studied by analyzing the evolution of Gaussian wave packet at different parameters and spatio-temporal evolution of the current on each bond of the graph. The model can have practical application in different systems arising in condensed matter physics and polymers. In condensed matter, the realization of the model comes from the fact that crystal lattices can be considered as branched structures, where the atoms in the lattice nodes exhibit harmonic vibrations. Such structure can be considered as a network of quantum harmonic oscillators. Very attractive application of the model is related to charge carrier transport in conducting polymers [32-38]. These latter can be considered as a network, where each branch is affected by external confining potential, which makes particle motion localized. Then the charge transport along such a network of potential wells will be reduced to its traveling from one well to another one. 


\section{References}

[1] Pauling L. The Diamagnetic Anisotropy of Aromatic Molecules, J. Chem. Phys., 1936, 4, P. 673.

[2] Ruedenberg K. and Scherr C.W. Free-Electron Network Model for Conjugated Systems. I. Theory, J. Chem. Phys., 1953,21 P. 1565.

[3] Exner P. and Seba P. Free quantum motion on a branching graph. Rep. Math. Phys., 1989, 28(1) P. 26.

[4] Kostrykin R. and Schrader R. Kirchhoff's rule for quantum wires. J. Phys. A: Math. Gen., 1999, 32, P. 595.

[5] Hul O., Bauch S., Pakonski P., Savytskyy N., Zyczkowski K. and Sirko L. Experimental simulation of quantum graphs by microwave networks, Phys. Rev. E, 2004, 69, P. 056205.

[6] Kottos T. and Smilansky U. Periodic Orbit Theory and Spectral Statistics for Quantum Graphs. Ann.Phys., 1999, 274(1), P. 124.

[7] Gnutzmann S. and Smilansky U. Quantum graphs: Applications to quantum chaos and universal spectral statistics. Adv.Phys., 2006, 55(5-6) P. 527-625.

[8] Gnutzmann S., Keating J.P. and Piotet F. Eigenfunction statistics on quantum graphs. Ann.Phys., 2010, 325(12) P. 2595-2640.

[9] Goldman N. and Gaspard P. Quantum graphs and the integer quantum Hall effect, Phys. Rev. B, 2008, 77, P. 024302.

[10] Russo A., Barnes E., Economou S.E. Photonic graph state generation from quantum dots and color centers for quantum communications, Phys. Rev. B, 2018, 98, P. 085303.

[11] Bolte J. and Harrison J. The spin contribution to the form factor of quantum graphs. J. Phys. A: Math. Gen., 2003,36, P. L433.

[12] Sabirov K., Yusupov J., Jumanazarov D., Matrasulov D. Bogoliubov de Gennes equation on metric graphs. Phys. Lett. A, 2018,382 , P. 2856.

[13] Giacomelli G., Lepri S., Trono C. Optical networks as complex lasers, Phys. Rev. A, 2019, 99, P. 023841.

[14] Yusupov J.R., Sabirov K.K., Ehrhardt M. and Matrasulov D.U. Transparent quantum graphs. Phys. Lett. A, 2019, 383, P. 2382.

[15] Aripov M.M., Sabirov K.K., Yusupov J.R. Transparent vertex boundary conditions for quantum graphs: simplified approach. Nanosystems: physics, chemistry, mathematics, 2019, 10(5), P. 501-602.

[16] Matrasulov D.U., Sabirov K.K. and Yusupov J.R. PT-symmetric quantum graphs. J. Phys. A: Math. Gen., 2019,52 , P. 155302.

[17] Yusupov J., Dolgushev M., Blumen A. and Mülken O. Directed transport in quantum star graphs. Quantum Inf Process, 2016,15, P. 1765.

[18] Yusupov J. Tunable wave packet transport in branched periodic lattices with time-dependent external field. Nanosystems: physics, chemistry, mathematics, 2017, 8(1), P. 42-47.

[19] Naimark K. and Solomyak M. Eigenvalue estimates for the weighted Laplacian on metric trees. Proc. London Math. Soc., 2000, 80, P. 690.

[20] Keating J.P. Fluctuation statistics for quantum star graphs. Quantum graphs and their applications. Contemp. Math., 2006, 415, P. 191-200.

[21] Harrison J.M., Smilansky U. and Winn B. Quantum graphs where back-scattering is prohibited. J. Phys. A: Math. Gen., 2007,40 , P. 14181.

[22] Exner P. and Lipovský J. On the absence of absolutely continuous spectra for Schrödinger operators on radial tree graphs. J. Math. Phys., 2010, 51, P. 122107.

[23] Berkolaiko G., Kuchment P. Introduction to Quantum Graphs, Mathematical Surveys and Monographs, AMS, 2013.

[24] Mugnolo D. Semigroup Methods for Evolution Equations on Networks. Springer-Verlag, Berlin, 2014.

[25] Kurasov P., Ogik R. and Rauf A. On reflectionless equi-transmitting matrices. Opuscula Math., 2014,34 , P. 483.

[26] P. Exner and H. Kovarik, Quantum waveguides. Springer, 2015.

[27] Joly P., Kachanovska M. and Semin A. Wave propagation in fractal trees. Mathematical and Numerical Issues. hal-01801394, 2018.

[28] Yusupov J.R., Sabirov K.K., Ehrhardt M. and Matrasulov D.U. Transparent nonlinear networks. Phys. Rev. E, 2019 , 100, P. 032204.

[29] Aquino N. The isotropic bounded oscillators. J. Phys. A: Math. Gen., 1997, 30(7) P. 2403.

[30] Stevanović L. and Sen K.D. Eigenspectrum properties of the confined 3D harmonic oscillator. J. Phys. B: At.Mol., 2008,41 P. 225002.

[31] Amore P. and Fernández F.M. One-dimensional oscillator in a box. Eur. J. Phys., 2010, 31, P. 69.

[32] Rice M.J., Gartstein Yu.N. Excitons and Interband Excitations in Conducting Polymers Based on Phenylene. Phys. Rev. Lett., 1994, 73, P. 2504.

[33] Brazovskii S., Kirova N., Bishop A.R. Theory of electronic states and excitations in PPV. Opt. Mat., 1998,9 P. $465-471$.

[34] Moses D., Wang J., Heeger A.J., Kirova N., Brazovski S. Electric field induced ionization of the exciton in poly(phenylene vinylene). Synth. Met., 2001, 119, P. 503.

[35] Kirova N., Brazovskii S. Optical and electrooptical absorption in conducting polymers. Thin Solid Films, 2002,403, P. 419.

[36] Kirova N., Brazovskii S. Conjugated polymers at the verge of strongly correlated systems and 1d semiconductors, Synth. Met., 2004, 141, P. 139.

[37] Kirova N., Brazovskii S. Electronic interactions and excitons in conducting polymers, Current Appl. Phys., 2004,4 P. $473-478$.

[38] Kobrak M.N., Bittner E.R. A dynamic model for exciton self-trapping in conjugated polymers. I. Theory, J. Chem. Phys., 2000,112 P. 5399. 